\title{
La tesis de título de Guillermo Ulriksen Becker (1952): Bases para la Planeación Regional del Norte Chico: Provincias de Atacama y Coquimbo.
}

\author{
María Isabel Pávez Reyes
}

Bases para la Planeación Regional del Norte Chico: Provincias de Atacama y Coquimbo, -tesis de título en planeamiento regional de Guillermo Ulriksen Becker (n.1905 - m. 1979) presentada en 1953 en la, entonces, "Facultad de Arquitectura" de la Universidad de Chile-, constituye no sólo una radiografía del estado del desarrollo del Norte Chico hace cincuenta y cinco años, sino que también un señalamiento del potencial posible de desarrollar en la perspectiva del largo plazo, y de los aspectos negativos que se van sucediendo o pudieran suceder atentando contra dicho potencial.

El Arqto. Ulriksen, quien ha sido testigo y actor excepcional en el Norte Chico a fines de los años 40 y comienzos de los 50, ofrece una mirada descriptiva y explicativa original y crítica, de los diversos sectores de la economía, sin olvidar las costumbres observadas en la población, y la riqueza de la flora y fauna encontrada en sus exhaustivos recorridos por el Norte Chico de Chile. Estos conocimientos y experiencias han tenido lugar en circunstancias de su trabajo -conjunto con el paisajista alemán Oscar Prager- para efectos del proyecto del llamado "Plan Serena" desde 1947, primero, y de su tesis de título de Arquitecto en 1952-1953, a continuación. En esta última, intenta aportar bases para la planeación regional del Norte Chico, a partir de las carencias que él estima existieron en el mandato del "Plan Serena".

Guillermo Ulriksen fue alumno Karl Brunner en los años 1930 en Facultad de Arquitectura de la Universidad de Chile, institución donde cursó la carrera de arquitectura. Fue activista de la Reforma de 1945; ayudante y colaborador del Arqto. Hector Mardones Restat en el concurso y edificación del premiado proyecto del edificio del Banco del Estado; pionero de la enseñanza técnica integrada de los cursos de instalaciones sanitarias en los talleres de Arquitectura y Urbanismo - una línea no exenta de dificultades teniendo en cuenta sus propuestas estéticas y su avanzado criterio rupturista para introducir, con los maestros de la "Bioarquitectura", los todavía nuevos paradigmas neo-ecólogicos-. También fue un delicado pintor de paisajes, y un destacado atleta. Cabría agregar la visión territorial que desarrollaba en la última etapa de su búsqueda creativa como investigador, plasmada, entre otros ejemplos, en sus estudios sobre Aysén y Valparaíso siguiendo la línea que introdujera el Arqto. Luis Muñoz Maluschka y que continuara, con variada suerte, el Arqto. Juan Parrochia Beguin $^{1}$.

En lo referido a su trabajo en el "Plan Serena", podemos señalar que si la Corporación de Reconstrucción y Auxilio no había podido cumplir su tarea en el territorio que originó su existencia en ocasión del terremoto de 1939, el Estado la hizo participar activamente desde 1947 -junto con otros organismos técnicos y económicos- en un intento de Planeación Regional impulsado directamente desde el Gobierno ${ }^{2}$.

En efecto, el Presidente Gabriel González Videla (1946-1952), había expuesto en un documento publicado en 1951 con el nombre de "Plan de Fomento y Urbanización para

\footnotetext{
1 Conceptos aportados por el Arqto. Prof. Alberto Gurovich W., ex alumno de Guillermo Ulriksen, en entrevista realizada por la autora en mayo de 2008.

2 URBINA VERDUGO, René. 1953, Profesor del IVUP, [inédito] Facultad de Arquitectura, U. Chile, La Planeación en Chile, informe, Santiago de Chile, marzo de 1953, 4 p.
} 
las Provincias de Chile", lo que Ilamó "Plan Experimental de Urbanización", y que se concretó especialmente en "Plan Serena"3.

Argumentando que las grandes carencias en las ciudades del norte y sur de Chile y, el desmedido crecimiento de Santiago, le habían inducido a impulsar la reforma constitucional de descentralización económica y administrativa del país, y a tomar la responsabilidad personal de intervenir en la planificación urbanística y económica de las provincias para poner "término definitivo" a la migración hacia Santiago, señaló los fundamentos, medios y formas de realización de sus ideas y proyectos.

Se había seleccionado la Provincia de Coquimbo y su capital, La Serena, porque esta era, históricamente, la segunda ciudad de Chile, fundada dos años después que Santiago. Su planta se había concebido "genialmente" en la orilla de un río y a corta distancia del océano Pacífico, teniendo cerca el puerto de Coquimbo. Por ello, era más fácil que en cualquiera otra capital de provincia, el "ensayo de transformación urbanística y estética de la ciudad". Se devolvería a la ciudad su clásico estilo arquitectónico ("colonial") y su primitiva perspectiva del océano y del valle, lo cual se estimó de interés local y nacional ${ }^{4}$.

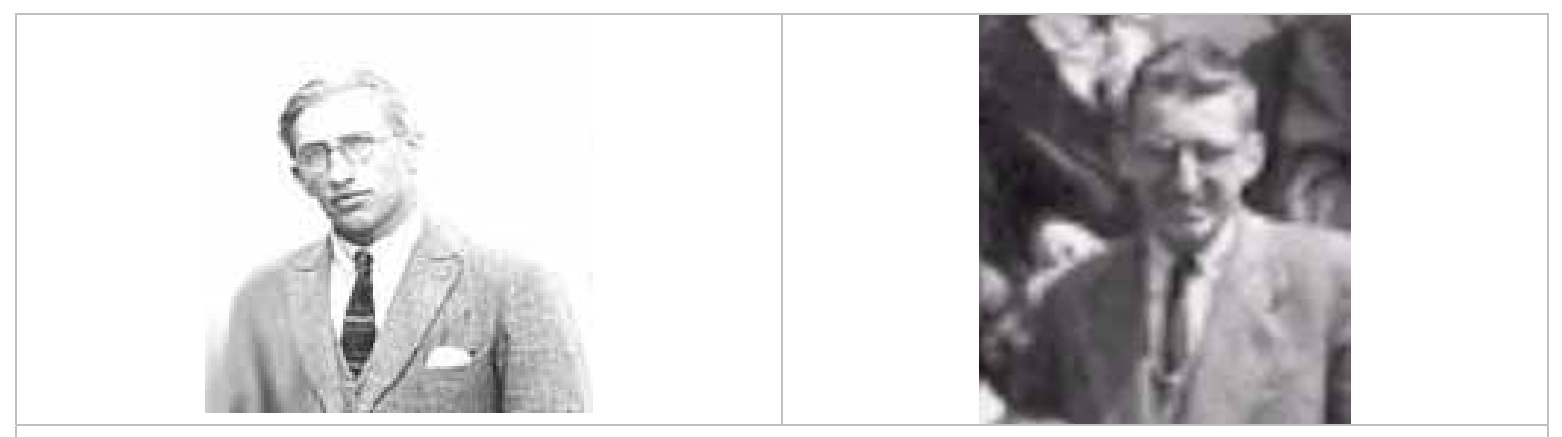

ARQTO. F. GUILLERMO ULRIKSEN BECKER (n. Concepción, 1905 - m. Santiago de Chile, 1979), ex alumno y ex profesor de la Facultad de Arquitectura, en Santiago y Valparaíso, de la Universidad de Chile. Co-autor, conjuntamente con el paisajista Oscar Prager, del "Plan Serena". Fte.: Archivos fotográficos de la F.A.U. de la Universidad de Chile.

Este Plan estuvo a cargo del Arqto. Ulriksen (Arqto. E), conjuntamente con el paisajista alemán residente en el país, Oscar Prager, quien fue su amigo, a pesar de no ser totalmente afines en su pensamiento. Para conseguir los objetivos económicos y estilísticos que singularizaron este Plan, el propio Presidente impuso muchas directrices, movilizando, además, a casi todas las entidades de fomento y acción técnica del país para la realización de las obras de regadío, forestación, colonización extranjera, vialidad, activación de yacimientos mineros y otros.

Cabe destacar que el "Plan Serena" motivó fuertes reacciones en los círculos políticos y técnicos, y especialmente en las restantes provincias de Chile, las que estimaron

${ }^{3}$ CHILE, PRESIDENCIA DE LA REPÚBLICA. Plan de Fomento y Urbanización para las Provincias de Chile, 1951.

${ }^{4}$ Señaló el Presidente que fueron todas esas razones, y no sólo "la exaltación sentimental del hijo que ama a su tierra natal", lo que lo decidió a elegir La Serena para desarrollar el plan experimental que debía servir de guía a las otras provincias del país. (CHILE, PRESIDENCIA DE LA REPÚBLICA. 1951, op. cit.). 
menoscabados sus intereses por la gran concentración de esfuerzos y recursos en La Serena.

En lo referido a planificación regional, las realizaciones consistieron en la recuperación de 3.000 hectáreas de terrenos pantanosos; la mecanización del puerto de Coquimbo, y la construcción del tramo de la Carretera Panamericana (Ruta 5 Norte, 1946) desde La Serena hasta Santiago (480 Kms.) ${ }^{5}$.

En cuanto a planificación urbana, una proposición formalista para La Serena incluyó una zonificación de usos del suelo, la restauración arquitectónica y la construcción de edificios educacionales y otros servicios públicos de la ciudad, 3.000 viviendas y 74 hectáreas de parques nuevos. Destacó la voluntad de habilitar los centros de las manzanas -conforme a las enseñanzas de Brunner-, para hacerlos más permeables a los peatones y vehículos, sin embargo, esto ese logró en la realidad sólo en una manzana, y parcialmente ${ }^{6}$.

Confirmamos la limitación del abordaje regional del plan toda vez que, en 1952-53, el mismo Guillermo Ulriksen, se vio motivado a realizar su tesis antes citada para optar finalmente al título de Arquitecto ${ }^{7}$.

Ulriksen justifica su tema de tesis, señalando que para establecer las bases científicas del planeamiento de una ciudad es necesario conocer previamente la "trastierra" (hinterland), esto es, la geografía económica de la región que rodea a la ciudad ya existente. Era obvia para Ulriksen la necesidad de conocer la historia de la ciudad misma y también la historia del país ${ }^{8}$.

Esta tentativa de establecer bases para el planeamiento del Norte Chico, se plantea como un aporte personal del autor, como fruto de los contactos con los

\footnotetext{
${ }^{5}$ UNIVERSIDAD DE CHILE, FACULTAD DE ARQUITECTURA, I.V.U.P., 1958. Chile en el V Congreso de la Unión Internacional de Arquitectos 1958. Construcción y reconstrucción de ciudades, 29 págs. ilus., ingléscastellano.

${ }^{6}$ Ibidem.

7 ULRIKSEN B., Guillermo. 1952. Bases para la Planeación Regional del Norte Chico. Provincias de Atacama y Coquimbo (Región de los Valles Transversales), Tesis de Título, Arquitectura, Facultad de Arquitectura y Urbanismo [sic], Santiago de Chile, Prof. Guía Arqto. Santiago AGUIRRE del C., julio de 1952.

La memoria de esta tesis comprende estudios y reflexiones sobre la demografía, la migración, la estadística agrícola y ganadera, sobre el carácter del comercio interior y exterior de las provincias de Atacama y Coquimbo -que el autor reconoció como conjunto regional, "Región de los Valles Transversales"-, sobre la renta, sobre los proyectos de regadío, sobre la experiencia "en sentido positivo y negativo" en la realización del "Plan de Fomento y Urbanización de las Provincias de Chile" aplicado al Norte Chico, y especialmente a la ciudad de La Serena; sobre la flora del Norte Chico, y otros.

Ulriksen lamentó no haber alcanzado a tratar en su tesis algunos problemas muy importantes:

"Uno de los problemas que no he alcanzado a analizar es el de la hoya del Salado, considerada como territorio autárquico dependiente enteramente de la minería y en elevadísimo porcentaje, de la explotación de los minerales de cobre de Potrerillos. Otro tema que hubiera deseado desarrollar es el de las ciudades del Norte Chico, de los problemas específicamente urbanos (no urbanísticos) de Copiapó, Vallenar, Coquimbo, Andacollo, Ovalle, Combarbalá, Illapel, Salamanca y Los Vilos. Entre centenares de cuestiones, podría haber dejado constancia de que Los Vilos no está unido a la Red Telefónica Nacional; que cuenta con una oficina de telégrafos atendida con un horario restringido, no obstante ser un importante centro de comunicaciones marítimo, ferroviario, y últimamente, terrestre. Hubiera deseado hacer un balance de los déficit urbanísticos de La Serena; pero en especial, de la enorme subestimación de sus satélites suburbanos como Compañía Baja (3.000 habitantes) y Compañía Alta ( 1.000 habitantes). Habría podido incluir un análisis de los tipos de viviendas obreras empleados por las compañías mineras; ilustrar la vivienda típica de EL TOFO con su entresuelo ultra económico o 'cabrete' donde duerme hacinada la familia, penetrando a dicho recinto por una 'escalera de gallinero' ".

(Ibidem, p. PREFACIO 1-2).
} 
problemas más candentes de la región. Sostengo que no puede elaborarse un planeamiento claro sin una finalidad clara. La finalidad del planeamiento sólo puede emanar de un plan perfectamente claro de desarrollo de la economía que consulte, como una parte de él, la nacionalización de las explotaciones mineras en manos extranjeras $\mathrm{y}$, además, una profunda reforma agraria, conjuntamente con medidas de orden financiero, educacional, etc. De ahí que esta tesis se limitará a estudiar las bases para un planeamiento regional. Sostengo, además, que una Junta de Planeamiento no podría formular un plan para el Norte Chico con la perspectiva de algunos años, porque durante la elaboración de ese plan se descubrirán grandes lagunas en el conocimiento científico de esa región lo cual impedirá la formulación de ese plan regional. El autor se propone señalar algunas lagunas en el conocimiento científico de esa región, que pudo constatar en su labor de seis años en el terreno y a través de las discusiones en el seno de pequeñas reuniones de planeamiento. El autor tratará de formular aquellas deducciones de sus observaciones que complementen el conocimiento actual de la geografía, demografía, biología, producción y desarrollo de los recursos naturales de esta región. También ha de repetir nociones elementales sobre dichos temas, pero tratará de ilustrar esas nociones conocidas con ejemplos nuevos. Tiene el propósito de restringir el informe dándole el carácter de una aportación complementaria al conjunto de observaciones y conocimientos que autores tan versados como Fuenzalida, Vila, Matthey, Opazo, Reiche, Brueggen, Fenner, Monsalve, Montalva, Bennett, Harnecker, Ochoa, Zepeda, Frei, Ocampo, Videla Lira, Benítez, Caro y Chouteau, han señalado ya como característico de la geografía económica del Norte Chico. En las conclusiones, el autor espera poder superar esa categoría de aportación complementaria y lograr formular un programa sencillo que sirva de 'aide-memoire' de los futuros planificadores del Norte Chico". (...)

Señala también Ulriksen las dificultades que encontraba el planeamiento en Chile en la época9 :

No se ha creado un organismo planificador nacional que coordine las iniciativas de la Corporación de Fomento de la Producción, de la Empresa Nacional de Electricidad, de la Corporación de la Vivienda, de la Corporación de Inversiones y otros organismos que hacen planificaciones parciales. Por lo menos deberían realizarse anualmente congresos o conferencias regionales y nacionales de planeación en forma sistemática; pero, como es sabido, ni siquiera se realizan en el país y en el continente congresos conjuntos de ingenieros, arquitectos y economistas. No se han echado aún las bases para un movimiento nacional y continental pro-planificación.

No están ausentes en el trabajo de Guillermo Ulriksen, las críticas a la labor de planificación urbana previa en Chile:

Los planificadores deben tener el conocimiento directo de la región (práctica) y deben estudiar las bases científicas (teoría). Sin conocimiento personal de la geografía, población, agricultura, ganadería, minería, comunicaciones y transportes de una región, ningún planificador podrá ser capaz de elaborar un Plano Regulador Urbano. Exclusivamente en base de Geografía Física se han elaborado en nuestro país los planos reguladores urbanos. Sobre aquella base fue elaborado hace veinte años el

\footnotetext{
${ }^{9}$ Ibidem, p. "Regadío" - 9. Por otra parte, refirió a modo de ejemplo, diez proyectos sectoriales en curso en la región del Norte Chico, carentes de toda coordinación. Observó también la necesidad de crear una cartoteca nacional, un archivo nacional de proyectos, y una oficina para el inventario del utillaje tecnológico nacional (sondas de prospección, equipos fotográficos, pantógrafos de precisión y otros). Tampoco existía una legislación adecuada para lograr la coordinación de los proyectos que permitiera alcanzar, en el futuro, una planificación nacional de todos los recursos naturales, e iniciativas conducentes a la explotación científica de ellos. (Ibidem, p. "Regadío"-1).
} 
Plano Regulador de la Comuna de Santiago ["Plano Oficial de Urbanización de la Comuna de Santiago", 1934/39, Karl BRUNNER]. Dieciocho años transcurridos desde su promulgación han evidenciado que las previsiones de este plano eran precarias. La expansión real de la ciudad amenaza de asfixia a la Comuna de Santiago. Es sabido que no se estableció ni siquiera un esquema del plan del futuro desarrollo de la vialidad en la región de Santiago en aquella oportunidad; de ahí el actual embotellamiento en la Carretera Panamericana en su acceso norte a Santiago. La experiencia de la Planificación demuestra que habría sido más fácil establecer un buen plan regional de Santiago, hace veinte años, que un buen plan urbano; el grado de exactitud es más difícil de establecer a medida que se restringe el área de planeamiento. Ello se debe a que los fenómenos económicos se densifican en el mismo grado en que crece la densidad de la población; las mallas del planeamiento se hacen más complejas. De ahí la importancia de las tentativas que se hagan para establecer en primer lugar Planes Regionales.

Cabe destacar por último que, para el período 1929-1949, después de las tesis del Arqto. Luis Muñoz Maluschka, "Nuevas poblaciones en territorio de colonización" (Alemania, 1931), y "Concepción dinámica del Urbanismo: Espacios Vitales Urbanos" (Chile, 1940) aplicada a la zona devastada por el terremoto de 1939, se podría reconocer en el presente trabajo de titulación de Guillermo Ulriksen -más que en el "Plan Serena"-, una tercera tesis de aproximación a una planificación territorial en Chile en el siglo $\mathrm{XX}$, en la perspectiva integradora de los diversos objetivos sectoriales que es necesario atender.

En la perspectiva de preservar y difundir el patrimonio intelectual de nuestra Facultad de Arquitectura y Urbanismo, nos complace presentar en la sección Memoria Histórica de Revista de Urbanismo N¹8, la edición electrónica de esta tesis, a partir de la copia matriz que se encuentra en la Biblioteca Central de la Facultad de Arquitectura y Urbanismo de la Universidad de Chile.

Completamos esta breve presentación con un listado bibliográfico de algunos de los escritos publicados o editados por el Arqto. Guillermo Ulriksen B. ${ }^{10}$

Santiago, mayo de 2008.

\section{Algunos escritos y publicaciones de Guillermo Ulriksen Becker:}

1968. ULRIKSEN BECKER, GUILLERMO. Dos Hipótesis de Expansión de Valparaíso Chile, Valparaíso, 83 págs.

1963. ULRIKSEN BECKER, Guillermo; GÓMEZ, Sergio; PÉREZ L., Octavio. "La cooperación técnica mutua", (ponencias de congresos) publicado en: Comité Asesor del Consejo de Rectores, Instituto Chileno de Administración Racional de Empresas; Cámara Chilena de la Construcción, 1963, 6 p., Jornadas Universidad - Empresa de la Construcción, Primeras, Chile, Santiago, agosto 26 al 28, 1963.

1962. ULRIKSEN BECKER, Guillermo (Arquitecto del Instituto de Vivienda Urbanismo y Planeación de la Facultad de Arquitectura de la Universidad de Chile), 1962. La Gestación de los Planes Reguladores Urbanos en Chile en el período 1940-1960. Impreso en la Escuela de Arquitectura de la Universidad de Chile. (Extracto referido a Osorno en Revista de Urbanismo digital $\mathrm{N}^{\circ} 11$, dic. 2004.) http://revistaurbanismo.uchile.cl

${ }^{10}$ La autora de esta presentación, y editora responsable de la versión electrónica de la Tesis de Título de Guillermo Ulriksen Becker es Dra. Arquitecta en la FAU de la U. de Chile, Editora de Revista de Urbanismo y Curadora del Patrimonio Cultural de la FAU. U. de Chile. 
1959. ULRIKSEN BECKER, Guillermo. "Ensayo de método para la agrupación de bloques de viviendas en conformidad a disposiciones del Reglamento Especial de Viviendas Económicas" [ponencias de congresos]. 1959. Publicado en: Algunos estudios de los aspectos urbanísticos del Plan Habitacional impulsado por el Supremo Gobierno en 1959. Universidad de Chile, Facultad de Arquitectura, Instituto de Vivienda Urbanismo y Planeación.

1952. ULRIKSEN BECKER, Guillermo. Bases para la Planeación Regional del Norte Chico. Provincias De Atacama \& Coquimbo (Región de los Valles Transversales). Tesis de Título de Arquitecto, Facultad de Arquitectura de la Universidad de Chile, Prof. Guía: Sr. Santiago Aguirre del C. (Versión digital completa PDF- enlace desde Revista de Urbanismo $N^{\circ} 18,2008$.

http://revistaurbanismo.uchile.cl

1952. ULRIKSEN BECKER, Guillermo. Observaciones complementarias sobre la Flora del Norte Chico. Provincias de Atacama y Coquimbo o Región de los Valles Trasversales de Chile. (Escrito unitario, Anexo de su tesis de título, en versión digital en Revista de Urbanismo digital N¹8, 20 págs.)

http://revistaurbanismo.uchile.cl

1952. ULRIKSEN BECKER, Guillermo, responde entrevista a J. Torres Valencia: "Plan Constructivo La Serena - Coquimbo". El Mercurio, Santiago, 7 marzo 1952. pág. 27. (Se emitió indicar el nombre del entrevistado, según informa él mismo en su tesis de título citada). (En versión digital en Revista de Urbanismo digital $\left.\mathrm{N}^{\circ} 18\right)$.

http://revistaurbanismo.uchile.cl

1947. ULRIKSEN BECKER, Guillermo. Darwin y su compañero Mariano González, 1947. [Darwin fue huésped de campesinos pobres del Norte Chico, independientes, ..."lo que no es común en Chile"... comentaba Darwin)].

1943. ULRIKSEN BECKER, Guillermo. ¿Cómo aumentar la producción agrícola?. El Estado debe impulsar el empleo de salitre. S/d.

1943. ULRIKSEN BECKER, Guillermo. Hacia la planeación regional en Chile, 1943. (Publicado, según Informa el autor en su tesis de título citada, en varias revistas -[s/d]-, y divulgado por el autor a través de varios programas radiales, 6 págs., multicopiadas).

\section{Otro antecedente:}

En noviembre de 2004, el Centro de ex Alumnos de la Escuela de Arquitectura de la Universidad de Chile, Sede Valparaíso, y de la Universidad de Valparaíso, realizaron un homenaje a G. Ulriksen B., en reunión de camaradería. 